\title{
Zastavnyi Operators and Positive Definite Radial Functions
}

\author{
Tarik Faouzi ${ }^{\mathrm{a}}$, Emilio Porcu ${ }^{\mathrm{b}, *}$, Moreno Bevilacqua $^{\mathrm{c}}$, Igor Kondrashuk ${ }^{\mathrm{d}}$ \\ ${ }^{a}$ Department of Statistics, Applied Mathematics Research Group, University of Bio Bio, Chile. \\ ${ }^{b}$ School of Mathematics and Statistics, University of Newcastle, UK. Department of Mathematics and \\ University of Atacama, Copiapó, and Millennium Nucleus Center for the Discovery of Structures in \\ Complex Data, Chile. \\ ${ }^{c}$ University of Valparaiso, Department of Statistics. Millennium Nucleus Center for the Discovery of \\ Structures in Complex Data, Chile. \\ ${ }^{d}$ Grupo de Matemática Aplicada, Departamento de Ciencias Básicas, Universidad del B io-B io, Campus \\ Fernando May, Av. Andres Bello 720, Casilla 447, Chillán, Chile
}

\begin{abstract}
we consider a new operator acting on rescaled weighted differences between two members of the class $\Phi_{d}$ of positive definite radial functions. In particular, we study the positive definiteness of the operator for the Matérn, Generalized Cauchy and Wendland families.

Keywords: Completely Monotonic, Fourier Transforms, Positive Definite, Radial Functions
\end{abstract}

\section{Introduction}

Positive definite functions are fundamental to many branches of mathematics as well as probability theory, statistics and machine learning amongst others. There has been an increasing interest in positive definite functions in $d$-dimensional Euclidean spaces ( $d$ is a positive integer throughout), and the reader is referred to [7], [14], [13], [17] and [16].

This paper is concerned with the class $\Phi_{d}$ of continuous functions $\phi:[0, \infty) \mapsto \mathbb{R}$ such that $\phi(0)=1$ and the function $\boldsymbol{x} \mapsto \phi(\|\boldsymbol{x}\|)$ is positive definite in $\mathbb{R}^{d}$. The class $\Phi_{d}$ is nested, with the strict inclusion relation:

$$
\Phi_{1} \supset \Phi_{2} \supset \cdots \supset \Phi_{d} \supset \cdots \supset \Phi_{\infty}:=\bigcap_{d \geq 1} \Phi_{d}
$$

The classes $\Phi_{d}$ are convex cones that are closed under product, non-negative linear combinations, and pointwise convergence. Further, for a given member $\phi$ in $\Phi_{d}$, the rescaled function $\phi(\cdot / \alpha)$ is still in $\Phi_{d}$ for any given $\alpha$. We make explicit emphasis on this fact because it will be repeatedly used subsequently.

\footnotetext{
${ }^{*}$ Corresponding author.

Email addresses: tfaouzi@ubiobio.cl (Tarik Faouzi), emilio.porcu@newcastle.ac.uk (Emilio Porcu ), Moreno.bevilacqua@uv.cl (Moreno Bevilacqua), igor .kondrashuk@ubiobio.cl (Igor Kondrashuk)
} 
For any nonempty set $A \subseteq \mathbb{R}^{d}$, we call $C(A)$ the set of continuous functions from $A$ into $\mathbb{R}$. For $p$ a positive integer, let $\boldsymbol{\theta} \in \Theta \subset \mathbb{R}^{p}$ and let $\{\phi(\cdot ; \boldsymbol{\theta}), \boldsymbol{\theta} \in \Theta\}$ be a parametric family belonging to the class $\Phi_{d}$. For $\varepsilon \in \mathbb{R}, \varepsilon \neq 0$ and $0<\beta_{1}<\beta_{2}$ with $\beta_{i}, i=1,2$ two scaling parameters, we define the Zastavnyi operator $K_{\varepsilon ; \boldsymbol{\theta} ; \beta_{2}, \beta_{1}}[\phi]: \Phi_{d} \mapsto C(\mathbb{R})$ by

$$
K_{\varepsilon ; \boldsymbol{\theta} ; \beta_{2}, \beta_{1}}[\phi](t)=\frac{\beta_{2}^{\varepsilon} \phi\left(\frac{t}{\beta_{2}} ; \boldsymbol{\theta}\right)-\beta_{1}^{\varepsilon} \phi\left(\frac{t}{\beta_{1}} ; \boldsymbol{\theta}\right)}{\beta_{2}^{\varepsilon}-\beta_{1}^{\varepsilon}}, \quad t \geq 0
$$

with $K_{\varepsilon ; \boldsymbol{\theta} ; \beta_{2}, \beta_{1}}[\phi](0)=1$. Here, by $\beta_{i}^{\varepsilon}$ we mean $\beta_{i}$ raised to the power of $\varepsilon$. It can be namely checked that

$$
K_{\varepsilon ; \boldsymbol{\theta} ; \beta_{2}, \beta_{1}}[\phi](0)=\frac{\beta_{2}^{\varepsilon} \phi\left(\frac{0}{\beta_{2}} ; \boldsymbol{\theta}\right)-\beta_{1}^{\varepsilon} \phi\left(\frac{0}{\beta_{1}} ; \boldsymbol{\theta}\right)}{\beta_{2}^{\varepsilon}-\beta_{1}^{\varepsilon}}=\frac{\beta_{2}^{\varepsilon}-\beta_{1}^{\varepsilon}}{\beta_{2}^{\varepsilon}-\beta_{1}^{\varepsilon}}=1
$$

A motivation for studying positive definiteness of the radial functions $\mathbb{R}^{d} \ni \mathbf{x} \mapsto$ $K_{\varepsilon ; \boldsymbol{\theta} ; \beta_{2}, \beta_{1}}[\phi](\|\mathbf{x}\|)$ comes from the problem of monotonicity of the so-called microergodic parameter of specific parametric families [4, 5] when studying the asymptotic properties of the maximum likelihood estimation under fixed domain asymptotics. The operator (1.1) is a generalization of the operator proposed in [12] where $\varepsilon$ is assumed to be positive. Our problem can be formulated as follows:

Problem 1.1. Let $d$ and $q$ be positive integers. Let $\left\{\phi(\cdot ; \boldsymbol{\theta}), \boldsymbol{\theta} \in \Theta \subset \mathbb{R}^{q}\right\}$ be a family of functions belonging to the class $\Phi_{d}$. Find the conditions on $\varepsilon \in \mathbb{R}, \varepsilon \neq 0$ and $\boldsymbol{\theta}$, such that $K_{\varepsilon ; \boldsymbol{\theta} ; \beta_{2}, \beta_{1}}[\phi]$ as defined through (1.1) belongs to the class $\Phi_{n}$ for some $n=1,2, \ldots$ for given $0<\beta_{1}<\beta_{2}$.

We first note that Problem 1.1 has at least two possible solutions. Indeed, direct inspection shows

$$
\lim _{\varepsilon \rightarrow+\infty} K_{\varepsilon ; \boldsymbol{\theta} ; \beta_{2}, \beta_{1}}[\phi](t)=\phi\left(\frac{t}{\beta_{1}} ; \boldsymbol{\theta}\right), \quad \lim _{\varepsilon \rightarrow-\infty} K_{\varepsilon ; \boldsymbol{\theta} ; \beta_{2}, \beta_{1}}[\phi](t)=\phi\left(\frac{t}{\beta_{2}} ; \boldsymbol{\theta}\right), \quad t \geq 0,
$$

where the convergence is pointwise in $t$.

The positive definiteness of (1.1), assuming $\varepsilon>0$, has been studied in [12] when $\phi$ belongs to the Buhmann class [6]. An important special case of the Buhmann class is the the Generalized Wendland family [8]. For $\kappa>0$, we define the class $\mathcal{G W}:[0, \infty) \rightarrow \mathbb{R}$ as:

$$
\mathcal{G W}(t ; \kappa, \mu)= \begin{cases}\frac{\int_{t}^{1} u\left(u^{2}-t^{2}\right)^{\kappa-1}(1-u)^{\mu} \mathrm{du}}{B(2 \kappa, \mu+1)}, & 0 \leq t<1 \\ 0, & t \geq 1\end{cases}
$$

and, for $\kappa=0$, by continuity we have

$$
\mathcal{G W}(t ; 0, \mu)= \begin{cases}(1-t)^{\mu}, & 0 \leq t<1 \\ 0, & t \geq 1\end{cases}
$$


The function $\mathcal{G} \mathcal{W}(t ; \kappa, \mu)$ is a member of the class $\Phi_{d}$ if and only if $\mu \geq 0.5(d+1)+\kappa[18$ ]. [12] found that if $\phi(\cdot ; \boldsymbol{\theta})=\mathcal{G} \mathcal{W}(\cdot ; \kappa, \mu)$ and $\varepsilon>0$ then $K_{\varepsilon ; \kappa, \mu ; \beta_{2}, \beta_{1}}[\mathcal{G W}](t)$ is positive definite if $\mu \geq(d+7) / 2+\kappa$ and $\epsilon \geq 2 \kappa+1$.

This paper is especially interested to the solution of Problem 1.1 when considering two celebrated parametric families:

The Matérn family. In this case, $\phi(\cdot ; \boldsymbol{\theta})=\mathcal{M}(\cdot ; \nu)$, so that the $\boldsymbol{\theta}=\nu$, a scalar and $\Theta=(0, \infty)$, with

$$
\mathcal{M}(t ; \nu)=\frac{2^{1-\nu}}{\Gamma(\nu)} t^{\nu} \mathcal{K}_{\nu}(t), \quad t \geq 0,
$$

where $\mathcal{K}_{\nu}$ is the modified Bessel function of the second kind of order $\nu>0$ [1]. The functions $\mathcal{M}(\cdot ; \nu), \nu>0$, belong to the class $\Phi_{\infty}[15]$.

The Generalized Cauchy family. In this case $\phi(\cdot ; \boldsymbol{\theta})=\mathcal{C}(\cdot ; \delta, \lambda)$, so that $\boldsymbol{\theta}=(\delta, \lambda)^{\top}$, with $\top$ denoting the transpose operator. Here, $\Theta=(0,2] \times(0, \infty)$, and

$$
\mathcal{C}(t ; \delta, \lambda)=\left(1+t^{\delta}\right)^{-\lambda / \delta}, \quad t \geq 0 .
$$

The functions $\mathcal{C}(\cdot ; \delta, \lambda)$ belong to the class $\Phi_{\infty}[9]$.

Additionally, we provide a solution to Problem 1.1 when $\phi(\cdot ; \boldsymbol{\theta})=\mathcal{G} \mathcal{W}(\cdot ; \kappa, \mu), \boldsymbol{\theta}=(\kappa, \mu)^{\top}$, $\Theta=[0, \infty) \times(0, \infty)$, assuming $\varepsilon<0$.

To give an idea of how the operator $K_{\varepsilon ; \boldsymbol{\theta} ; \beta_{2}, \beta_{1}}[\phi](t)$ acts on $\phi(t, \boldsymbol{\theta})$ for given $0<\beta_{1}<\beta_{2}$ when $\phi$ is the Matérn family, Figure $1(\mathrm{~A})$ compares $K_{\varepsilon ; 0.5 ; \beta_{2}, \beta_{1}}[\mathcal{M}](t)$ with $\mathcal{M}\left(t / \beta_{1} ; 0.5\right)$ and $\mathcal{M}\left(t / \beta_{2} ; 0.5\right)$ when $\beta_{1}=0.075, \beta_{2}=0.15, \varepsilon=1$ (red line) and $\varepsilon=-2$ (blue line). We note that the behaviour at the origin of $K_{1 ; 0.5 ; \beta_{2}, \beta_{1}}[\mathcal{M}](t)$ changes drastically with respect to the behaviour at the origin of $\mathcal{M}(\cdot ; 0.5)$. Moreover, $K_{-2 ; 0.5 ; \beta_{2}, \beta_{1}}[\mathcal{M}](t)$ can attain negative values. It turns out from Theorem 3.1 that $K_{1 ; 0.5 ; \beta_{2}, \beta_{1}}[\mathcal{M}](t) \in \Phi_{\infty}$ and $K_{-2 ; 0.5 ; \beta_{2}, \beta_{1}}[\mathcal{M}](t) \in \Phi_{2}$.

A similar graphical representation is given in Figure 1 (B) when $\phi \equiv \mathcal{C}$, the Cauchy family in (1.5). In this case, we consider $\varepsilon=-0.7,1.25, \delta=0.6, \lambda=2.5, \beta_{1}=0.2, \beta_{2}=0.3$. Note that,under this setting, $K_{-1.25 ; 0.6,2.5 ; \beta_{2}, \beta_{1}}[\mathcal{C}](t)$ attains negative values as well. It turns out from from Theorem $3.3 K_{\varepsilon ; 2.5,0.6 ; \beta_{2}, \beta_{1}}[\mathcal{C}](t) \in \Phi_{\infty}$ for $\varepsilon=-0.7$ and 1.25 .

Finally, Figure $1(\mathrm{C})$ compares $K_{\varepsilon ; 0,4.5 ; \beta_{2}, \beta_{1}}[\mathcal{G W}](t)$ with $\mathcal{G W}\left(t / \beta_{1} ; 0,4.5\right)$ and $\mathcal{G W}\left(t / \beta_{2} ; 0,4.5\right)$ with $\beta_{2}=0.6, \beta_{1}=0.4$ when $\varepsilon=1$ (red line) and $\varepsilon=-2$ (blue line). As for the Matérn case, the behaviour at the origin of $K_{\varepsilon ; 0,4.5 ; \beta_{2}, \beta_{1}}[\mathcal{G W}](t)$ changes neatly with respect to the behaviour at the origin of $\mathcal{G W}(\cdot ; 0,4.5)$. Moreover $K_{-2 ; 0,4.5 ; \beta_{2}, \beta_{1}}[\mathcal{G W}](t)$ can reach negative values. It turns out from Theorem 3.2 that $K_{-2 ; 0,4.5 ; \beta_{2}, \beta_{1}}[\mathcal{G W}](t)$ belongs to $\Phi_{2}$.

These three examples show that operator (1.1) can change substantially the features of given families $\phi$ in terms of both differentiability at the origin and negative correlations. The solution of Problem 1.1 for the Matérn and Cauchy families, passes necessarily through the specification of the properties of the radial Fourier transforms of the radially symmetric functions $\mathcal{M}(\|\cdot\| ; \nu)$ and $\mathcal{C}(\|\cdot\| ; \delta, \lambda)$ in $\mathbb{R}^{d}$. For the Matérn family, such a Fourier transform is available in closed form. For the Generalized Cauchy family we obtain the Fourier transform as series expansions generalizing a recent result obtained by [11]. The plan of the paper is 


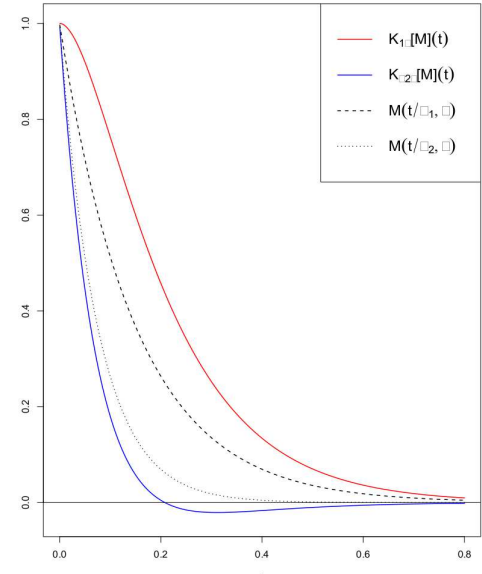

(A)

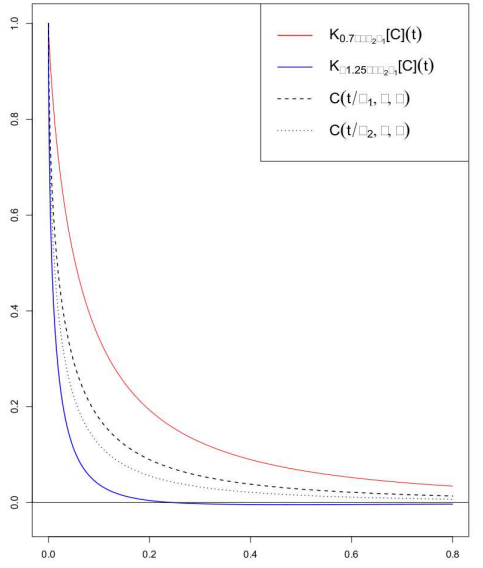

(B)

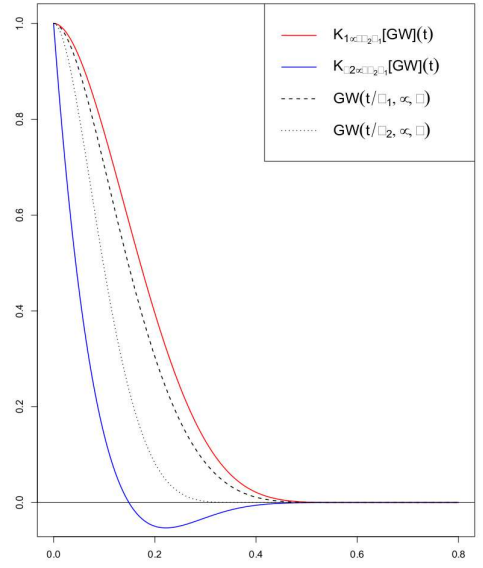

(C)

Figure 1: From left to right: (A) comparison of $K_{\varepsilon ; \nu ; \beta_{2}, \beta_{1}}[\mathcal{M}](t)$ when $\varepsilon=1$ (red line) and $\varepsilon=-2$ (blue line) with $\mathcal{M}\left(t / \beta_{1} ; \nu\right)$ and $\mathcal{M}\left(t / \beta_{2} ; \nu\right)$ when $\nu=0.5, \beta_{1}=0.075, \beta_{2}=0.15$. (B) comparison of $K_{\varepsilon ; \delta, \lambda ; \beta_{2}, \beta_{1}}[\mathcal{C}](t)$ when $\varepsilon=0.7$ (red line) and $\varepsilon=-1.25$ (blue line) with $\mathcal{C}\left(t / \beta_{1} ; \delta, \lambda\right)$ and $\mathcal{C}\left(t / \beta_{2} ; \delta, \lambda\right.$ ) when $\delta=0.6, \lambda=2.5$, $\beta_{1}=0.2, \beta_{2}=0.3$. (C) comparison of $K_{\varepsilon ; \mu, \kappa ; \beta_{2}, \beta_{1}}[\mathcal{G W}](t)$ when $\varepsilon=1$ (red line) and $\varepsilon=-2$ (blue line) with $\mathcal{G W}\left(t / \beta_{1} ; \mu, \kappa\right)$ and $\mathcal{G} \mathcal{W}\left(t / \beta_{2} ; \mu, \kappa\right)$ when $\mu=4.5, \kappa=0, \beta_{1}=0.4, \beta_{2}=0.6$.

the following: Section 2 contains the necessary preliminaries and background. Section 3 gives the main results of this paper.

\section{Preliminaries}

We start with some expository material. A function $f: \mathbb{R}^{d} \rightarrow \mathbb{R}$ is called positive definite if, for any collection $\left\{a_{k}\right\}_{k=1}^{N} \subset \mathbb{R}$ and points $\boldsymbol{x}_{1}, \ldots, \boldsymbol{x}_{N} \in \mathbb{R}^{d}$, the following holds:

$$
\sum_{k=1}^{N} \sum_{h=1}^{N} a_{k} f\left(\boldsymbol{x}_{k}-\boldsymbol{x}_{h}\right) a_{h} \geq 0 .
$$

By Bochner's theorem, continuous positive definite functions are the Fourier transforms of positive and bounded measures, that is

$$
f(\boldsymbol{x})=\int_{\mathbb{R}^{d}} \mathrm{e}^{\mathrm{i}\langle\boldsymbol{x}, \boldsymbol{z}\rangle} F(\mathrm{~d} \boldsymbol{z}), \quad \boldsymbol{x} \in \mathbb{R}^{d}
$$

where $\langle\cdot, \cdot\rangle$ denotes inner product and where $\mathrm{i}$ is the complex number such that $\mathrm{i}^{2}=-1$. Additionally, if $f(\boldsymbol{x})=\phi(\|\boldsymbol{x}\|)$ for some continuous function defined on the positive real line, Schoenberg's theorem [7, with the references therein] shows that $f$ is positive definite if and only if its radial part $\phi$ can be written as

$$
\phi(t)=\int_{[0, \infty)} \Omega_{d}(\xi t) G_{d}(\mathrm{~d} \xi), \quad t \geq 0
$$


where $G_{d}$ is a positive and bounded measure, and where

$$
\Omega_{d}(t)=t^{-(d-2) / 2} J_{(d-2) / 2}(t), \quad t \geq 0,
$$

where $J_{\nu}$ defines a Bessel function of order $\nu$. If $\phi(0)=1$, then $G_{d}$ is a probability measure [7]. Classical Fourier inversion in concert with Bochner's theorem shows that the function $\phi$ belongs to the class $\Phi_{d}$ if and only if it admits the representation (2.2), and this in turn happens if and only if the function $\widehat{\phi}_{d}:[0, \infty) \rightarrow[0, \infty)$, defined through

$$
\widehat{\phi}_{d}(z):=\mathcal{F}_{d}[\phi(t)](z)=\frac{z^{1-d / 2}}{(2 \pi)^{d}} \int_{0}^{\infty} t^{d / 2} J_{d / 2-1}(t z) \phi(t) \mathrm{d} t, \quad z \geq 0
$$

is nonnegative and such that $\int_{[0, \infty)} \widehat{\phi}_{d}(z) z^{d-1} \mathrm{~d} t<\infty$. Note that we intentionally put a subscript $d$ into $G_{d}$ and $\widehat{\phi}_{d}$ to emphasize the dependence on the dimension $d$ corresponding to the class $\Phi_{d}$ where $\phi$ is defined. This is explicitly stated in [7], where it is explained that for any member of the class $\Phi_{d}$ there exists at least $G_{1}, \ldots, G_{d}$ non negative bounded measures in the representation (2.2). Hence the term $d$-Schoenberg measures proposed therein. Finally, a convergence argument as much as in Schoenberg [14] shows that $\phi \in \Phi_{\infty}$ if and only if

$$
\phi(\sqrt{t})=\int_{[0, \infty)} \mathrm{e}^{-\xi t} G(\mathrm{~d} \xi), \quad t \geq 0,
$$

for $G$ positive, nondecreasing and bounded. Thus, $\psi:=\phi(\sqrt{ } \cdot)$ is the Laplace transform of $G$, which shows, in concert with Bernstein's theorem, that the function $\psi$ is completely monotonic on the positive real line, that is $\psi$ is infinitely often differentiable and $(-1)^{k} \psi^{(k)}(t) \geq 0$, $t>0, k \in \mathbb{N}$. Here, $\psi^{(k)}$ denotes the $k$-th order derivative of $\psi$, with $\psi^{(0)}=\psi$ by abuse of notation.

For a given $d \in \mathbb{N}$, direct inspection shows that for any scale parameter $\beta>0$, the Fourier transform (2.3) of $\phi(\cdot / \beta)$ is identically equal to $\beta^{d} \widehat{\phi}_{d}(\beta \cdot)=: \widehat{\phi}_{d, \beta}(\cdot)$, and we shall repeatedly make use of this fact for the results following subsequently. It is well known that the Fourier transform of the Matérn covariance function $\mathcal{M}$ in Equation (1.4) is given by [15]

$$
\widehat{\mathcal{M}}_{d, \beta}(z ; \nu)=\frac{\Gamma(\nu+d / 2)}{\pi^{d / 2} \Gamma(\nu)} \frac{\beta^{d}}{\left(1+\beta^{2} z^{2}\right)^{\nu+d / 2}}, \quad z \geq 0 .
$$

An ingenious approach in [10] shows that the Fourier transform of Generalized Cauchy covariance function $\mathcal{C}$ in Equation (1.5) can be written as

$$
\widehat{\mathcal{C}}_{d, \beta}(z ; \delta, \lambda)=-\frac{\beta^{d / 2+1} z^{-d}}{2^{d / 2-1} \pi^{d / 2+1}} \Im\left(\int_{0}^{\infty} \frac{\mathcal{K}_{(d-2) / 2}(\beta t)}{\left(1+e^{i \frac{\pi \delta}{2}}(t / z)^{\delta}\right)^{\lambda / \delta}} t^{d / 2} \mathrm{~d} t\right), \quad z>0
$$

where $\Im$ denotes the imaginary part of a complex argument, $\mathcal{K}_{(d-2) / 2}$ is the modified Bessel function of the second kind of order $(d-2) / 2$ and $\beta$ is a scale parameter. A closed form expression for $\widehat{\mathcal{C}}_{d, \beta}$ has been elusive for longtime. [11] shed some light for this problem giving 
a infinite series representation of the spectral density under some specific restriction of the parameters. The result following below shows that the series representation given in [11] is valid without any restriction on the parameters.

Theorem 2.1. Let $\mathcal{C}(\cdot ; \delta, \lambda)$ be the Generalized Cauchy covariance function as defined in Equation (1.5). Then, it is true that

$$
\begin{aligned}
\widehat{\mathcal{C}}_{d, \beta}(z ; \delta, \lambda) & =\frac{z^{-d}}{\pi^{d / 2}} \frac{1}{\Gamma\left(\frac{\lambda}{\delta}\right)} \sum_{n=0}^{\infty} \frac{(-1)^{n}}{n !} \frac{\Gamma\left(\frac{\lambda}{\delta}+n\right) \Gamma\left(d / 2-\left(\frac{\lambda}{\delta}+n\right) \delta / 2\right)}{\Gamma\left(\left(\frac{\lambda}{\delta}+n\right) \delta / 2\right)}\left(\frac{z \beta}{2}\right)^{\left(\frac{\lambda}{\delta}+n\right) \delta} \\
& +\frac{z^{-d}}{\pi^{d / 2} \delta} \frac{2}{\Gamma\left(\frac{\lambda}{\delta}\right)} \sum_{n=0}^{\infty} \frac{(-1)^{n}}{n !} \frac{\Gamma\left(\frac{2 n+d}{\delta}\right) \Gamma\left(\frac{\lambda}{\delta}-\frac{2 n+d}{\delta}\right)}{\Gamma(n+d / 2)}\left(\frac{z \beta}{2}\right)^{2 n+d}
\end{aligned}
$$

with $z>0$, where $\delta \in(0,2)$ and $\lambda>0$.

Proof. The Mellin-Barnes transform is defined through the identity which is given by

$$
\frac{1}{(1+x)^{\alpha}}=\frac{1}{2 \pi \mathrm{i}} \frac{1}{\Gamma(\alpha)} \oint_{\Lambda} x^{u} \Gamma(-u) \Gamma(\alpha+u) \mathrm{d} u,
$$

here $\Gamma(\cdot)$ denotes the Gamma function. This representation is valid for any $x \in \mathbb{R}$. The contour $\Lambda$ contains the vertical line which passes between left and right poles in the complex plane $u$ from negative to positive imaginary infinity, and should be closed to the left in case $x>1$, and to the right complex infinity if $0<x<1$.

We now proceed to compute $\widehat{\mathcal{C}}_{d, \beta}$ as follows:

$$
\begin{aligned}
\widehat{\mathcal{C}}_{d, \beta}(\|\boldsymbol{z}\| ; \delta, \lambda) & =\mathcal{F}_{d}\left[\mathcal{C}_{d, \beta}(t ; \delta, \lambda)\right](\|\boldsymbol{z}\|) \\
& =\beta^{d} \mathcal{F}_{d}\left[\mathcal{C}_{d}(t ; \delta, \lambda)\right](\beta\|\boldsymbol{z}\|), \quad \boldsymbol{z} \in \mathbb{R}^{d} .
\end{aligned}
$$

Applying Equation (2.8), we obtain

$$
\begin{aligned}
\widehat{\mathcal{C}}_{d, \beta}(\|\boldsymbol{z}\| ; \delta, \lambda) & =\frac{\beta^{d}}{(2 \pi)^{d}} \frac{1}{\Gamma\left(\frac{\lambda}{\delta}\right)} \int_{\mathbb{R}^{d}} \mathrm{e}^{\mathrm{i} \beta\langle\boldsymbol{z}, \boldsymbol{x}\rangle} \oint_{\Lambda} \Gamma(-u) \Gamma\left(u+\frac{\lambda}{\delta}\right)\|\boldsymbol{x}\|^{u \delta} \mathrm{d} u \mathrm{~d} \boldsymbol{x} \\
& =\frac{\beta^{d}}{(2 \pi)^{d}} \frac{1}{\Gamma\left(\frac{\lambda}{\delta}\right)} \oint_{\Lambda} \Gamma(-u) \Gamma\left(u+\frac{\lambda}{\delta}\right) \int_{\mathbb{R}^{d}} \mathrm{e}^{\mathrm{i} \beta\langle\boldsymbol{z}, \boldsymbol{x}\rangle}\|\boldsymbol{x}\|^{u \delta} \mathrm{d} \boldsymbol{x} \mathrm{d} u .
\end{aligned}
$$

We now invoke the well known relationship [2],

$$
\int_{\mathbb{R}^{d}} \mathrm{e}^{\mathrm{i}\langle\boldsymbol{z}, \boldsymbol{x}\rangle}\|\boldsymbol{x}\|^{u \delta} \mathrm{d} \boldsymbol{x}=\frac{2^{d+u \delta} \pi^{d / 2} \Gamma(d / 2+u \delta / 2)}{\Gamma(-u \delta / 2)\|\boldsymbol{z}\|^{d+u \delta}},
$$

and, by abuse of notation, we now write $z:=\|\boldsymbol{z}\|$. We have

$$
\begin{aligned}
\widehat{\mathcal{C}}_{d, \beta}(z ; \delta, \lambda) & =\frac{\beta^{d}}{(2 \pi)^{d}} \frac{1}{\Gamma\left(\frac{\lambda}{\delta}\right)} \frac{1}{2 \pi \mathrm{i}} \oint_{\Lambda} \Gamma(-u) \Gamma\left(u+\frac{\lambda}{\delta}\right) \frac{2^{d+u \delta} \pi^{d / 2} \Gamma(d / 2+u \delta / 2)}{\Gamma(-u \delta / 2)} \frac{1}{(\beta z)^{d+u \delta}} \mathrm{d} u \\
& =\frac{z^{-d}}{\pi^{d / 2}} \frac{1}{\Gamma\left(\frac{\lambda}{\delta}\right)} \frac{1}{2 \pi \mathrm{i}} \oint_{\Lambda} \frac{\Gamma(-u) \Gamma\left(u+\frac{\lambda}{\delta}\right) \Gamma(d / 2+u \delta / 2)}{\Gamma(-u \delta / 2)}\left(\frac{2}{z \beta}\right)^{u \delta} \mathrm{d} u
\end{aligned}
$$


For any given value of $|2 / z \beta|$, it does not matter whether it is smaller or greater than 1 . In fact, we may close the contour to the left complex infinity. This is a convergent series for any values of the variable $z$ because we have a situation when denominator supresses numerator in the coefficient in front of powers of $z$. We now observe that the functions $u \mapsto \Gamma\left(\frac{\lambda}{\delta}+u\right)$ and $u \mapsto \Gamma\left(d / 2+\frac{u \delta}{2}\right)$ contain poles in the complex plane, respectively when $\frac{\lambda}{\delta}+u=-n$, and when $d / 2+\frac{u \delta}{2}=-n, n \in \mathbb{N}$. Using this fact and through direct inspection we obtain that the right hand side in (2.9) matches with (2.7). The proof is completed.

\section{Main Results}

We start by providing a solution to Problem 1.1 when $\phi(\cdot ; \boldsymbol{\theta})=\mathcal{M}(\cdot ; \nu)$, so that $\boldsymbol{\theta} \equiv \nu$ and $\Theta=(0, \infty)$.

Theorem 3.1. Let $\mathcal{M}(\cdot ; \nu)$ be the Matérn function as defined in Equation (1.4). Let $K_{\varepsilon ; \nu ; \beta_{2}, \beta_{1}}[\mathcal{M}]$ with $0<\beta_{1}<\beta_{2}$, be the Zastavnyi operator (1.1) related to the function $\mathcal{M}(\cdot ; \nu)$.

1. Let $\varepsilon>0$. Then, $K_{\varepsilon ; \nu ; \beta_{2}, \beta_{1}}[\mathcal{M}] \in \Phi_{\infty}$ if and only if $\varepsilon \geq 2 \nu>0$;

2. For a given $d \in \mathbb{N}$, let $\varepsilon<0$. Then, $K_{\varepsilon ; \nu ; \beta_{2}, \beta_{1}}[\mathcal{M}] \in \Phi_{d}$ if and only if $\varepsilon \leq-d<0$.

The following result gives some conditions for the solution of Problem 1.1 when $\phi(\cdot ; \boldsymbol{\theta})=$ $\mathcal{G W}(\cdot ; \mu, \kappa)$, so that $\boldsymbol{\theta}=(\kappa, \mu)^{\top}, \Theta=[0, \infty) \times(0, \infty)$.

Theorem 3.2. Let $d$ be a positive integer. Let $\mathcal{G W}(\cdot ; \mu, \kappa)$ be the function defined through Equations (1.2) and (1.3), for $\kappa>0$ and $\kappa=0$ respectively. Let $K_{\varepsilon ; \mu, \kappa, \beta_{2}, \beta_{1}}[\mathcal{G W}]$ with $0<\beta_{1}<\beta_{2}$, be the Zastavnyi operator (1.1) related to the function $\mathcal{G W}(\cdot ; \mu, \kappa)$. Then:

1. If $\varepsilon \geq 2 \kappa+1>0$ and $\mu \geq(d+7) / 2+\kappa$, then $K_{\varepsilon ; \mu, \kappa, \beta_{2}, \beta_{1}}[\mathcal{G W}] \in \Phi_{d}$.

2. $K_{\varepsilon ; \mu, \kappa, \beta_{2}, \beta_{1}}[\mathcal{G W}] \in \Phi_{d}$ if and only if $\varepsilon=2 \kappa+1$ and $\mu \geq(d+7) / 2+\kappa$.

3. If $\varepsilon \leq-d<0$ and $\mu \geq(d+7) / 2+\kappa$, then $K_{\varepsilon ; \mu, \kappa, \beta_{2}, \beta_{1}}[\mathcal{G W}] \in \Phi_{d}$.

4. $K_{-d ; \mu, \kappa, \beta_{2}, \beta_{1}}[\mathcal{G W}] \in \Phi_{d}$ if and only if $\mu \geq(d+1) / 2+\kappa$.

We now assume that for a fixed $d, \lambda>d$. This condition is necessary to ensure integrability of Generalized Cauchy covariance functions, and hence the related spectral density to be bounded. The following result provides a solution to Problem 1.1 when $\phi(\cdot ; \boldsymbol{\theta})=\mathcal{C}(\cdot ; \delta, \lambda)$ for $0<\delta<2$, so that $\Theta=(0,2) \times(d, \infty)$.

Theorem 3.3. Let $\mathcal{C}(\cdot ; \delta, \lambda)$ for $0<\delta<2$ be the Generalized Cauchy function as defined in Equation (1.5). Let $K_{\varepsilon ; \delta, \lambda ; \beta_{2}, \beta_{1}}[\mathcal{C}]$ with $0<\beta_{1}<\beta_{2}$, be the Zastavnyi operator (1.1) related to the function $\mathcal{C}(\cdot ; \delta, \lambda)$.

1. Let $\varepsilon>0$. If $\varepsilon \geq \delta>0$ and $\delta<1$, then $K_{\varepsilon ; \delta, \lambda ; \beta_{2}, \beta_{1}}[\mathcal{C}] \in \Phi_{\infty}$;

2. Let $\varepsilon<0$. $K_{\varepsilon ; \delta, \lambda ; \beta_{2}, \beta_{1}}[\mathcal{C}] \in \Phi_{\infty}$ if and only if $\varepsilon \leq-\lambda<0$.

Two technical lemmas are needed prove our last result. 
Lemma 3.1. Let $\mathcal{K}_{\nu}:[0, \infty) \rightarrow \mathbb{R}$ be the function defined through (1.4). Let $\nu>0$. Then, for all $z>0$,

1. $\lim _{z \rightarrow+\infty} z \frac{\mathcal{K}_{\nu}^{\prime}(z)}{\mathcal{K}_{\nu}(z)}=-\infty$, for all $\nu \in(-\infty,+\infty)$;

2. $\lim _{z \rightarrow+0} z \frac{\mathcal{K}_{\nu}^{\prime}(z)}{\mathcal{K}_{\nu}(z)}=-\nu, \quad$ for $\nu>1$.

Proof. To prove the two assertions, it is enough to use the following result [3],

$$
-\sqrt{\frac{\nu}{\nu-1} z^{2}+\nu^{2}}<\frac{z \mathcal{K}_{\nu}^{\prime}(z)}{\mathcal{K}_{\nu}(z)}<-\sqrt{z^{2}+\nu^{2}},
$$

where the left hand side of (3.1) is true for all $\nu>1$, and the right hand side holds for all $\nu \in \mathbb{R}$.

Lemma 3.2. Let $\mathcal{C}(\cdot ; 2, \lambda)$ be the Cauchy correlation function as defined at (1.5)). Let $\beta>0$. Then, for $d>\lambda / 2+2$ and $2 \epsilon<-\lambda$ the following assertions are equivalent:

1. $\beta^{\epsilon} \widehat{\mathcal{C}}_{d, \beta}(z ; 2, \lambda)$ is decreasing with respect to $\beta$ on $[0,+\infty)$, for every $z, \lambda$;

2. $\beta^{\varepsilon+\frac{2 d+\lambda}{4}} \mathcal{K}_{\frac{2 d-\lambda}{4}}(\beta)$ is decreasing with respect to $\beta$ on $[0,+\infty)$, for every $z, \lambda$;

3. $\left(\varepsilon+\frac{2 d+\lambda}{4}\right)+\beta \frac{\mathcal{K}_{\frac{2 d-\lambda}{4}}^{\prime}(\beta)}{\frac{\mathcal{K}_{2 d-\lambda}(\beta)}{4}}<0, \beta \in[0, \infty)$.

Proof. Showing that $\beta^{\varepsilon} \widehat{\mathcal{C}}_{d, \beta}(z ; 2, \lambda)$ is decreasing with respect to $\beta$ is the same as showing that $\beta^{\varepsilon+\lambda / 4+d / 2} \mathcal{K}_{d / 2-\lambda / 4}(\beta)$ is decreasing. Point 2 of Lemma 3.2 holds if and only if

$$
\beta^{\varepsilon+\frac{2 d+\lambda}{4}-1}\left(\left(\varepsilon+\frac{2 d+\lambda}{4}\right)+\beta \frac{\mathcal{K}_{\frac{2 d-\lambda}{4}}^{\prime}(\beta)}{\mathcal{K}_{\frac{2 d-\lambda}{4}}(\beta)}\right)<0 .
$$

Applying point 2 of Lemma 3.1 and the fact that $\beta \mathcal{K}_{\frac{2 d-\lambda}{4}}^{\prime}(\beta) / \mathcal{K}_{\frac{2 d-\lambda}{4}}(\beta)$ is decreasing with respect to $\beta$, the three assertions of Lemma 3.2 are true if $2 d>\lambda+4$ and $2 \varepsilon<-\lambda$. The proof is completed.

We are now able to fix a solution to Problem 1.1 when $\phi(\cdot ; \boldsymbol{\theta})=\mathcal{C}(\cdot ; 2, \lambda)$, so that $\boldsymbol{\theta}=\nu$ and $\Theta=(0, \infty)$.

Theorem 3.4. Let $\mathcal{C}(\cdot ; 2, \lambda)$ be the Cauchy function as defined in Equation (1.5) and let $K_{\varepsilon ; 2, \lambda ; \beta_{2}, \beta_{1}}[\mathcal{C}]$ with $0<\beta_{1}<\beta_{2}$ be the Zastavnyi operator (1.1) related to the function $\mathcal{C}(\cdot ; 2, \lambda)$. Then, for $\lambda<2 d-4, K_{\varepsilon ; 2, \lambda ; \beta_{2}, \beta_{1}}[\mathcal{C}] \in \Phi_{d}$ provided $2 \varepsilon<-\lambda$.

Proof. We need to find conditions such that $K_{\varepsilon ; 2, \lambda ; \beta_{2}, \beta_{1}}[\mathcal{C}] \in \Phi_{d}$. This is equivalent to the following condition:

$$
\beta_{1}^{\varepsilon} \widehat{\mathcal{C}}_{d, \beta_{1}}(z ; 2, \lambda)-\beta_{2}^{\varepsilon} \widehat{\mathcal{C}}_{d, \beta_{2}}(z ; 2, \lambda) \geq 0 .
$$

Thus, we need to prove that the function $\beta^{\varepsilon} \widehat{\mathcal{C}}_{d, \beta}(z ; 2, \lambda)$ is decreasing with respect to $\beta$. Using Lemma 3.2, we have that $K_{\varepsilon ; 2, \lambda ; \beta_{2}, \beta_{1}}[\mathcal{C}] \in \Phi_{d}$. 


\section{Acknowledgements}

The authors dedicate this work to Viktor Zastavnyi for his sixtieth birthday.

Partial support was provided by Millennium Science Initiative of the Ministry of Economy, Development, and Tourism, grant "Millenium Nucleus Center for the Discovery of Structures in Complex Data" for Moreno Bevilacqua and Emilio Porcu, by FONDECYT grant 1160280, Chile for Moreno Bevilacqua and by FONDECYT grant 1130647 , Chile for Emilio Porcu and by grant Diubb 170308 3/I from the university of Bio Bio for Tarik Faouzi. Tarik Faouzi and Igor Kondrashuk thank the support of project DIUBB 172409 GI/C at University of Bío-Bío. The work of I.K. was supported in part by Fondecyt (Chile) Grants No. 1121030 and by DIUBB (Chile) Grant No. 1814093/R.

\section{References}

[1] Abramowitz, M. and Stegun, I. A., editors (1970). Handbook of Mathematical Functions. Dover, New York.

[2] Allendes, P., Kniehl, B. A., Kondrashuk, I., Notte-Cuello, E. A., and Rojas-Medar, M. (2013). Solution to Bethe-Salpeter equation via Mellin-Barnes transform. Nuclear Physics B, 870(1):243-277.

[3] Baricz, Á., Ponnusamy, S., and Vuorinen, M. (2011). Functional inequalities for modified Bessel functions. Expositiones Mathematicae, 29(4):399-414.

[4] Bevilacqua, M. and Faouzi, T. (2018). Estimation and prediction of Gaussian processes using Generalized Cauchy covariance model under fixed domain asymptotics. ArXiv e-prints.

[5] Bevilacqua, M., Faouzi, T., Furrer, R., and Porcu, E. (2018). Estimation and prediction using Generalized Wendland functions under fixed domain asymptotics. The Annals of Statistics. Forthcoming.

[6] Buhmann, M. D. (2001). A new class of radial basis functions with compact support. Math. Comput., 70(233):307-318.

[7] Daley, D. J. and Porcu, E. (2014). Dimension walks and Schoenberg spectral measures. Proc. Amer. Math. Soc., 142:1813-1824.

[8] Gneiting, T. (2002). Compactly supported correlation functions. Journal of Multivariate Analysis, 83:493-508.

[9] Gneiting, T. and Schlather, M. (2004). Stochastic Models that Separate Fractal Dimension and Hurst Effect. SIAM Rev., 46(2):269-282.

[10] Lim, S. and Teo, L. (2009). Gaussian fields and Gaussian sheets with Generalized Cauchy covariance structure. Stochastic Processes and Their Applications, 119(4):1325-1356.

[11] Lim, S. C. and Teo, L. P. (2010). Analytic and Asymptotic Properties of Multivariate Generalized Linnik's Probability Densities. Journal of Fourier Analysis and Applications, 16(5):715-747.

[12] Porcu, E., Zastavnyi, P., and Bevilacqua, M. (2017). Buhmann covariance functions, their compact supports, and their smoothness. Dolomites Research Notes on Approximation, 10:33-42.

[13] Schaback, R. (2011). The missing Wendland functions. Advances in Computational Mathematics, $34(1): 67-81$.

[14] Schoenberg, I. J. (1938). Metric spaces and completely monotone functions. Annals of Mathematics, pages $811-841$.

[15] Stein, M. L. (1999). Interpolation of Spatial Data. Some Theory of Kriging. Springer, New York.

[16] Wendland, H. (1995). Piecewise polynomial, positive definite and compactly supported radial functions of minimal degree. Advances in Computational Mathematics, 4:389-396.

[17] Wu, Z. (1995). Compactly supported positive definite radial functions. Advances in Computational Mathematics, 18(4):283-292.

[18] Zastavnyi, V. and Trigub, R. (2002). Positive definite splines of special form. English transl. in Sb. Math., 193:1771-1800. 